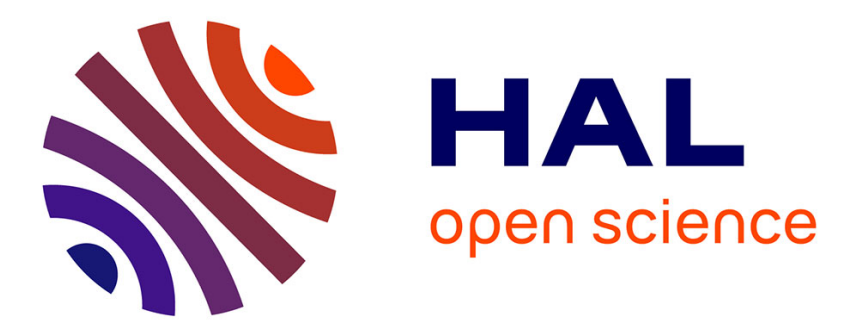

\title{
Interaction of few-optical-cycle solitons
}

Hervé Leblond, Igor V. Mel'Nikov, Dumitru Mihalache

\section{To cite this version:}

Hervé Leblond, Igor V. Mel'Nikov, Dumitru Mihalache. Interaction of few-optical-cycle solitons. Physical Review A: Atomic, molecular, and optical physics [1990-2015], 2008, 78 (4), pp.043802. 10.1103/PhysRevA.78.043802 . hal-03423700

\section{HAL Id: hal-03423700 \\ https://univ-angers.hal.science/hal-03423700}

Submitted on 10 Nov 2021

HAL is a multi-disciplinary open access archive for the deposit and dissemination of scientific research documents, whether they are published or not. The documents may come from teaching and research institutions in France or abroad, or from public or private research centers.
L'archive ouverte pluridisciplinaire $\mathbf{H A L}$, est destinée au dépôt et à la diffusion de documents scientifiques de niveau recherche, publiés ou non, émanant des établissements d'enseignement et de recherche français ou étrangers, des laboratoires publics ou privés. 


\title{
Interaction of few-optical-cycle solitons
}

\author{
H. Leblond \\ Laboratoire POMA, CNRS FRE 2988, Université d'Angers, 2 Boulevard Lavoisier, 49045 Angers Cedex 01, France \\ I. V. Mel'nikov \\ High Q Laboratories Inc., 2 Gledhill Crescent, Hamilton, Ontario, Canada L9C $6 H 4$ and Optolink Ltd., bldg 5, proezd 4806, \\ Zelenograd, 124498 Moscow, Russian Federation \\ D. Mihalache \\ Horia Hulubei National Institute for Physics and Nuclear Engineering (IFIN-HH), 407 Atomistilor, \\ Magurele-Bucharest 077125, Romania \\ (Received 23 July 2008; published 1 October 2008)
}

\begin{abstract}
By using the exact four-soliton solutions of the modified Korteweg-de Vries-sine Gordon equation describing the propagation of few-optical-cycle pulses in transparent media with instantaneous cubic nonlinearity, the interaction of two such initially well-separated pulses is studied. The shapes of soliton envelopes, the shifts in the location of envelope maxima, and the corresponding phase shifts are explicitly calculated.
\end{abstract}

DOI: 10.1103/PhysRevA.78.043802

PACS number(s): 42.65.Tg, 42.65.Re, 05.45.Yv

\section{INTRODUCTION}

In recent years, a considerable effort has been made in the field of ultrafast optics allowing the generation of ultraintense light pulses comprising merely a few optical cycles [1]. These ultrashort pulses have numerous applications in the field of light-matter interactions, high-order harmonic generation, extreme nonlinear optics [2], and attosecond physics [3].

Theoretical models involving corrections to the paraxial wave equations for the study of propagation of few-cycle pulses (FCPs) in nonlinear optical media such as fused silica [4] and photonic crystal fibers [5] have been developed during the past decade. Other theoretical approaches make use of the so-called "short-pulse equation," which is an integrable nonlinear partial differential equation describing the propagation of ultrashort optical pulses in media with cubic optical nonlinearities [6,7]. An issue of much interest from the application point of view is the pulse self-compression down to single cycle duration in transparent media with instantaneous cubic (Kerr) nonlinearity [8]. The propagation of FCPs in a self-focusing Kerr medium can be described beyond the slowly varying envelope approximation (SVEA) by using the modified Korteweg-de Vries (mKdV) [9], sineGordon (sG) [10,11], or $\mathrm{mKdV}-\mathrm{sG}$ equations [12]. The $\mathrm{mKdV}$ and $\mathrm{sG}$ equations are completely integrable by means of the inverse scattering transform method [13], whereas the $\mathrm{mKdV}-\mathrm{sG}$ equation is completely integrable only if some condition between its coefficients is satisfied [14]. All these equations admit breather solutions, which can realistically describe the few-optical-cycle solitons. In $(2+1)$ dimensions, the $\mathrm{mKdV}$ model is replaced by the (nonintegrable) generalized Kadomtsev-Petviashvili equation, which accounts for two-dimensional FCP soliton propagation [15,16]. Notice that, in quadratic nonlinear optical media, the FCP propagation is governed by the Korteweg-de Vries (KdV) equation, which does not admit breather solitons; in this case, halfcycle optical solitons in the form of a single hump with no oscillating tails would form [17].
It should be noted that the more general $\mathrm{mKdV}$-sG equation proved to describe fairly well the propagation of ultrashort optical pulses in a Kerr medium, thus demonstrating its remarkable mathematical capabilities in describing the physics of few-optical-cycle solitons [12]. Further, full numerical resolution of the Maxwell-Bloch equations for a three-level system [18] shows a good agreement with the results of the above asymptotic models. Because the $n$-soliton solution of the $\mathrm{mKdV}$-sG equation has been obtained before [19], we may easily get from it the four-soliton solution and, correspondingly, the two-breather solution, which can be used as the input in our study of the interaction between two FCPs. The two-breather solution describes the interaction in a Kerr medium of two few-optical-cycle solitons initially well separated, in any physical setting where one of the three integrable models mentioned above is a realistic one. Moreover, thanks to the existence of analytic expression for the two-breather solution, the location and phase shifts resulting from the interaction can be computed explicitly.

\section{FOUR-SOLITON AND TWO-BREATHER SOLUTIONS}

We consider the propagation of optical FCPs in a onedimensional self-focusing Kerr medium, such as a highly nonlinear optical fiber. In any physical application, FCPs will be periodically launched in the medium, in such a way that they propagate as solitons. Due to the fluctuations of the intensity of the laser source, the consecutive FCPs may have different power and energies, and consequently different velocities. Therefore, they are expected to cross each other. Our goal is to discuss what happens during the interaction.

The evolution of the electric field is governed by the $\mathrm{mKdV}$-sG equation, which reads in the completely integrable case as

$$
u_{\tau z}+a\left(\frac{3}{2} u_{\tau}^{2} u_{\tau \tau}+u_{\tau \tau \tau \tau}\right)-b \sin u=0
$$

where the dimensionless variables $u, z$, and $\tau$ are, respectively, proportional to the electric field, the propagation dis- 


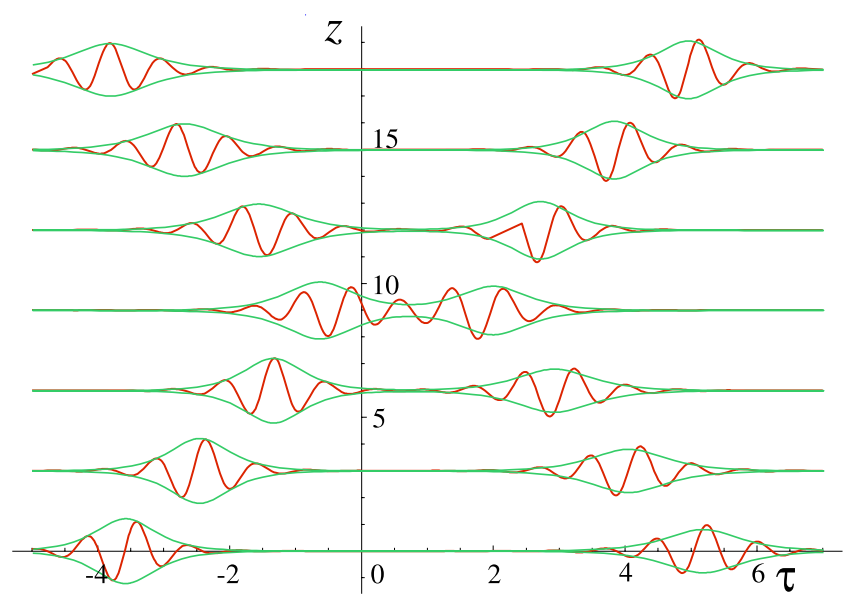

FIG. 1. (Color online) Interaction of two FCPs described by the two-breather (four-soliton) solution of $\mathrm{mKdV}-\mathrm{sG}$. The envelope is shown. Parameters: $p_{1}=2, p_{2}=2.5$, and $\omega_{1}=\omega_{2}=8$.

tance, and the retarded time, in a frame moving at the linear light velocity of the medium. Notice that $u$ is not an amplitude, but is proportional to the electric field itself. Both constants $a$ and $b$ are related to the dispersion and nonlinearity properties of the medium; see $[10,12]$. The integrable $\mathrm{mKdV}$-sG Eq. (1) reduces to the $\mathrm{mKdV}$ equation for $b=0$ and to the $\mathrm{sG}$ equation for $a=0$.

The $n$-soliton solution of the above $\mathrm{mKdV}$-sG equation is given by [19]

$$
u=2 i \ln \frac{f^{*}}{f},
$$

where

$$
f=\sum_{\mu_{j}=0,1} E_{\mu}
$$

with $\mu=\left(\mu_{1}, \ldots, \mu_{n}\right)$,

$$
E_{\mu}=\exp \left[\sum_{j=1}^{n} \mu_{j}\left(\xi_{j}+\frac{i \pi}{2}\right)+\sum_{l=1}^{n} \sum_{j=1}^{n} \mu_{j} \mu_{l} A_{j, l}\right],
$$

and

$$
\begin{gathered}
A_{j, l}=\ln \frac{\left(k_{j}-k_{l}\right)^{2}}{\left(k_{j}+k_{l}\right)^{2}}, \\
\xi_{j}=k_{j} \tau+\Omega_{j} z+\xi_{0 j}, \\
\Omega_{j}=\frac{b}{k_{j}}-a k_{j}^{3},
\end{gathered}
$$

$j, l=1, \ldots, n$ and the $k_{j}$ being arbitrary. These solutions are properly speaking solitons only for real $k_{j}$, but the solution holds algebraically for any complex $k_{j}$.

For $n=4$, and assuming

$$
k_{3}=k_{1}^{*}, \quad k_{4}=k_{2}^{*},
$$

we get the two-breather solution. We set below

$$
k_{1}=p_{1}+i \omega_{1}, \quad k_{2}=p_{2}+i \omega_{2},
$$

so that $\omega_{1}$ and $\omega_{2}$ are the characteristic frequencies of the two FCPs, and $1 / p_{1}$ and $1 / p_{2}$ are their durations.

\section{INPUT AND OUTPUT ENVELOPES}

The input and final breathers can be obtained as follows. Let us write $R=f^{*} / f$ as a function of the phases $\xi_{1}$ and $\xi_{2}$, and compute the limits

$$
\begin{aligned}
& R_{1 \pm}=\lim _{\xi_{2} \rightarrow \pm \infty} R, \\
& R_{2 \pm}=\lim _{\xi_{1} \rightarrow \pm \infty} R,
\end{aligned}
$$

with the $\xi_{j}$ defined as above. Then the quantities $u_{j \pm}$ $=2 i \ln R_{j \pm}(j=1,2)$ are the input and output isolated breathers.

The corresponding envelopes can be computed by replacing the varying part of the $\xi_{j}(j=1,2)$ by its real part, as

$$
\xi_{j} \leftarrow \xi_{r j}=p_{j} \tau+\Omega_{r j} z+d_{j}+i \varphi_{j}, \quad j=1,2,
$$

where

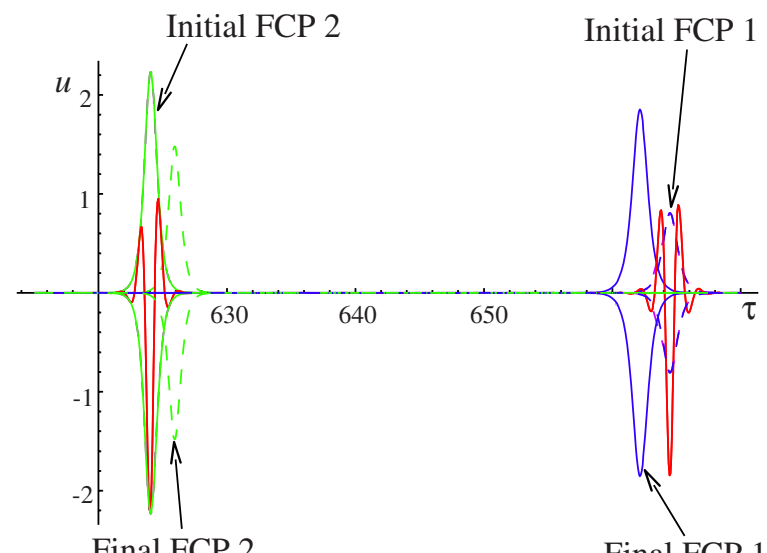

(a)

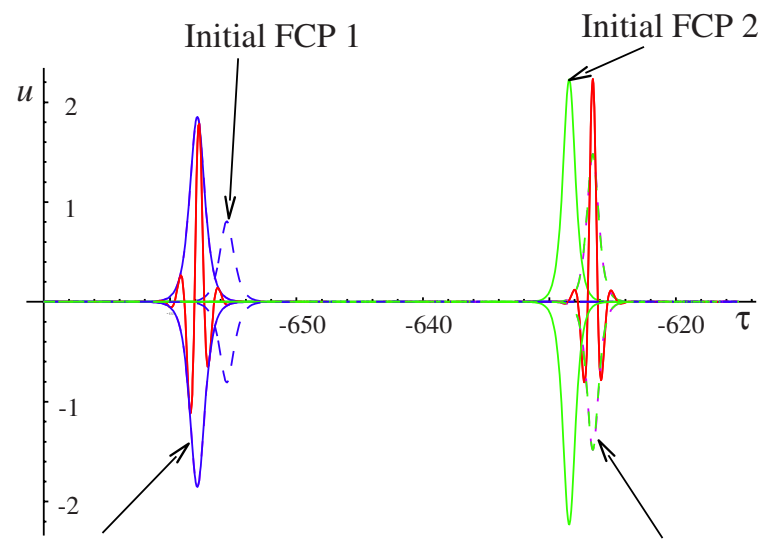

Final FCP 1

Final FCP 2

(b)

FIG. 2. (Color online) The FCPs and their initial and final envelopes before (a) and after (b) the interaction. Parameters: $p_{1}=2$, $p_{2}=2.5$, and $\omega_{1}=\omega_{2}=4$. 


$$
\begin{aligned}
& \Omega_{r j}=\operatorname{Re}\left(\omega_{j}\right), \\
& \xi_{0 j}=d_{j}+i \varphi_{j} .
\end{aligned}
$$

For an adequate choice of the constant phase $\varphi_{j}$, the value of $u$ obtained through this substitution is $u_{e}$, the envelope of $u$. We get in the same way the envelopes $u_{j \pm e}$ of the input and output breathers $u_{j \pm}$.

Figure 1 shows the general envelope. Figure 2(a) shows the two FCPs and their input and output envelopes, at $z$ $=-15$. Here the output envelopes are defined as the envelopes of the breather, at this propagation stage, which coincide with the output of the interaction as $z \rightarrow+\infty$, after having propagated alone. It is seen that the envelopes $u_{j+e}$, corresponding to the largest values of $\tau$, do not have the correct amplitude. The definition of the envelope implies indeed that the oscillation carrier is replaced by some constant $\varphi_{j}$ adequately chosen. In Fig. 2(a) [and also in Fig. 2(b)], this constant is taken as zero. It is the adequate value for the leftmost profiles $\left(u_{j-e}\right)$, not for the rightmost ones $\left(u_{j+e}\right)$, due to the arising of a phase shift during the interaction. This is discussed in detail in Sec. $\mathrm{V}$ below.

Figure 2(b) presents the same two-breather solution and input and output envelopes, at $z=+15$. The coincidence between the FCPs and envelopes, at least in location, is checked for the numerical values. The shift in the location of the breathers appears clearly in the figures.

\section{COMPUTATION OF THE LOCATION SHIFT}

Now we determine the location of the maxima of the envelopes. Precisely, we solve the equations $d R_{1 \pm} / d \xi_{1}=0$ and get the solutions $\xi_{m 1 \pm}$. Then the location shift is given by

$$
\delta \tau_{1}= \pm \frac{\xi_{m 1+}-\xi_{m 1-}}{p_{1}} .
$$

The sign depends on the relative velocity of the FCPs. The group velocity of the FCPs is computed as [12]

$$
w_{g j}=a\left(p_{j}^{2}-3 \omega_{j}^{2}\right)-\frac{b}{p_{j}^{2}+\omega_{j}^{2}}
$$

for $j=1,2$. In fact,

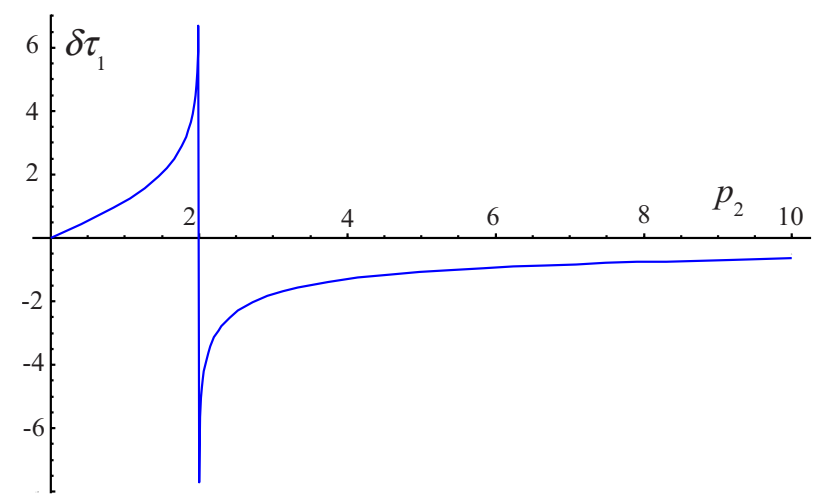

FIG. 3. (Color online) Variation of the location shift $\delta_{\tau_{1}}$ against the inverse duration $p_{2}$ of one pulse. Parameters: $p_{1}=2$ and $\omega_{1}$ $=\omega_{2}=4$.

$$
w_{g j}=\frac{-\Omega_{r j}}{p_{j}}
$$

$(j=1,2)$, i.e., $w_{g j}$ is the velocity of the characteristic variable $\xi_{r j}$. The variable $z$ is expressed in terms of the characteristic variables $\xi_{r 1}, \xi_{r 2}$ of the envelopes as

$$
z=\frac{\xi_{r 1} / p_{1}-\xi_{r 2} / p_{2}}{\Omega_{r 1} / p_{1}-\Omega_{r 2} / p_{2}},
$$

hence

$$
z \sim \frac{-1 / p_{2}}{\Omega_{r 1} / p_{1}-\Omega_{r 2} / p_{2}} \xi_{r 2}
$$

as $\xi_{r 2}$ tends to infinity, and $\xi_{r 2} \rightarrow \pm \infty$ corresponds to either $z \rightarrow+\infty$ or $z \rightarrow-\infty$ depending on the sign of

$$
\frac{-1 / p_{2}}{\Omega_{r 1} / p_{1}-\Omega_{r 2} / p_{2}},
$$

i.e., the sign of $\left[p_{2}\left(w_{g 1}-w_{g 2}\right)\right]$. Finally,

$$
\delta \tau_{1}=\operatorname{sgn}\left[p_{2}\left(w_{g 2}-w_{g 1}\right)\right] \frac{\xi_{m 1-}-\xi_{m 1+}}{p_{1}} .
$$

It yields

$$
\delta \tau_{1}=\operatorname{sgn}\left[p_{2}\left(w_{g 1}-w_{g 2}\right)\right] \times \frac{1}{p_{1}} \ln \left[\frac{\left[\left(\omega_{1}-\omega_{2}\right)^{2}+\left(p_{1}-p_{2}\right)^{2}\right]\left[\left(\omega_{1}+\omega_{2}\right)^{2}+\left(p_{1}-p_{2}\right)^{2}\right]}{\left[\left(\omega_{1}-\omega_{2}\right)^{2}+\left(p_{1}+p_{2}\right)^{2}\right]\left[\left(\omega_{1}+\omega_{2}\right)^{2}+\left(p_{1}+p_{2}\right)^{2}\right]}\right],
$$

and an analogue expression for $\delta_{\tau_{2}}$. Examples of the variations of $\delta_{\tau_{1}}$ against the FCP inverse duration $p_{2}$ and frequency $\omega_{2}$ are given in Figs. 3 and 4, respectively. It must be noticed that the shift $\delta_{\tau_{1}}$ does not depend on the coefficients $a, b$ of the $\mathrm{mKdV}$-sG equation, except its sign, via the group velocities. It becomes zero as soon as one of the pulses reaches the SVEA limit, which is obtained by setting either the inverse duration $p_{j}$ to zero or the frequency $\omega_{j}$ to infinity (see Figs. 3 and 4). An important feature is that, in contrast with the SVEA case, the interaction does not require any phase matching (nor any group velocity one). However there occurs a singularity in the case of two identical pulses. Since identical pulses have the same speed, they never cross together if they are well-separated initially. Two FCPs with 


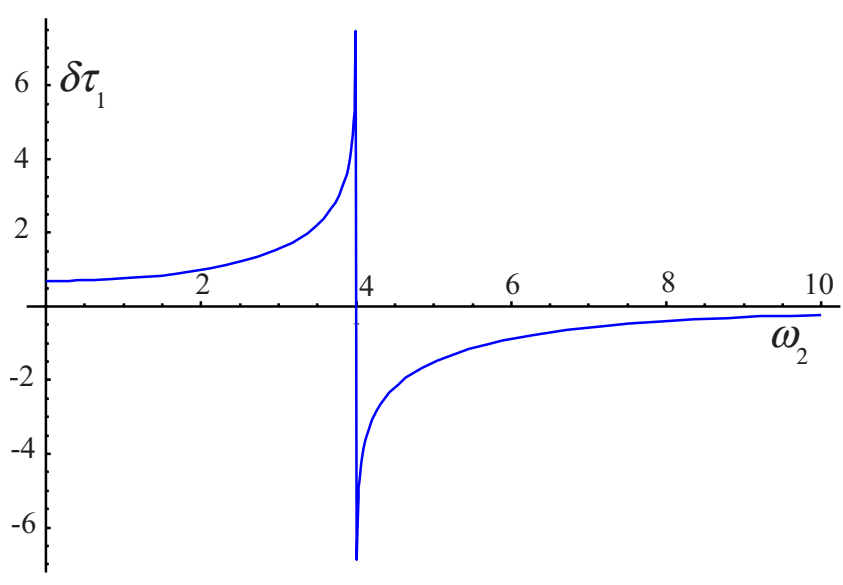

FIG. 4. (Color online) Variation of the location shift $\delta_{\tau_{1}}$ against the frequency $\omega_{2}$ of one pulse. Parameters: $p_{1}=p_{2}=2$ and $\omega_{1}=4$.

frequencies and durations close together will have velocities close together. If the propagation distance is large enough, they may interact over a long time. The above-mentioned singularity can be seen as a strong interaction for two pulses that are approximately matched in phase and group velocities.

\section{COMPUTATION OF THE PHASE SHIFT}

The phase shift $\delta_{\varphi_{1}}$, e.g., for the FCP 1, is computed by adjusting the phase $\varphi_{1}$ in such a way that the amplitude of the envelope $u_{1+e}$ is maximal (which yields the correct value of the amplitude of the envelope). The maximum is located

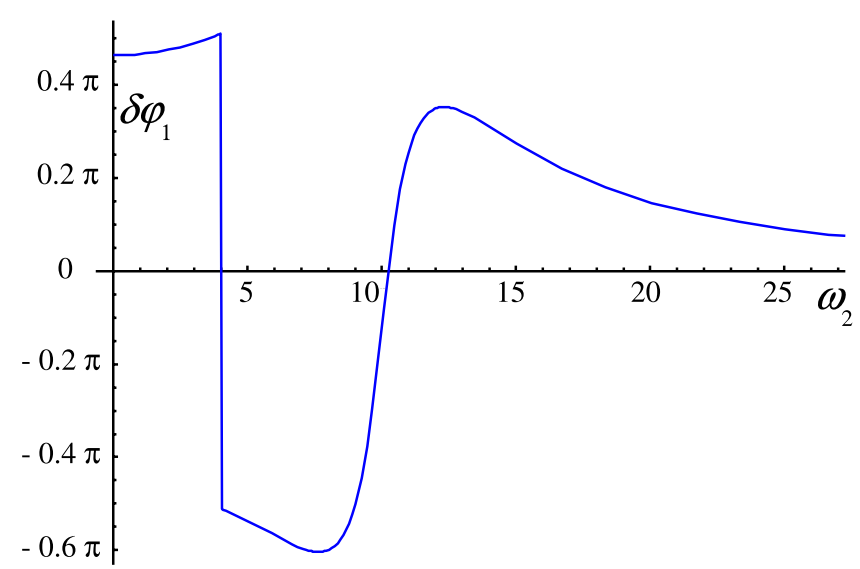

FIG. 6. (Color online) Same as Fig. 5, but for two FCPs with different durations. Parameters: $a=b=1, p_{1}=3, p_{2}=2$, and $\omega_{1}=10$.

at $x_{m 1+}=\xi_{m 1+} / p_{1}$. The peak value of the envelope is thus

$$
u_{m 1+}=\left.u_{1+e}\right|_{\xi_{r 1}}=\xi_{m 1+},
$$

and we only have to determine the value $\varphi_{m 1+}$ of the phase $\varphi_{1}$ which maximizes $u_{m 1+}$. In fact, it is easier to find the value $\varphi_{01+}$ of the phase $\varphi_{1}$ for which $u_{m 1+}$ is zero, then $\varphi_{m 1+}=\varphi_{01+} \pm \pi / 2$. The value of the phase that maximizes $u_{1-e}$ is always 0 . The relationship between the limits $z$ $\rightarrow \pm \infty$ and $\xi_{r 2} \rightarrow \pm \infty$ is the same as above, and hence the phase shift is given by

$$
\delta \varphi_{1}=\operatorname{sgn}\left[p_{2}\left(w_{g 1}-w_{g 2}\right)\right] \varphi_{m 1+} .
$$

The phase shift is explicitly computed to yield

$$
\delta \varphi_{1}=\operatorname{sgn}\left[p_{2}\left(w_{g 1}-w_{g 2}\right)\right] \times 2 \arctan \left[\frac{4 \omega_{1} p_{2}\left(\omega_{2}^{2}-\omega_{1}^{2}+p_{2}^{2}-p_{1}^{2}\right)}{\left(\omega_{1}^{2}-\omega_{2}^{2}\right)^{2}+\left(p_{1}^{2}-p_{2}^{2}\right)^{2}+2\left(\omega_{1}^{2}+\omega_{2}^{2}\right)\left(p_{1}^{2}+p_{2}^{2}\right)-8 \omega_{1}^{2} p_{2}^{2}}\right] .
$$

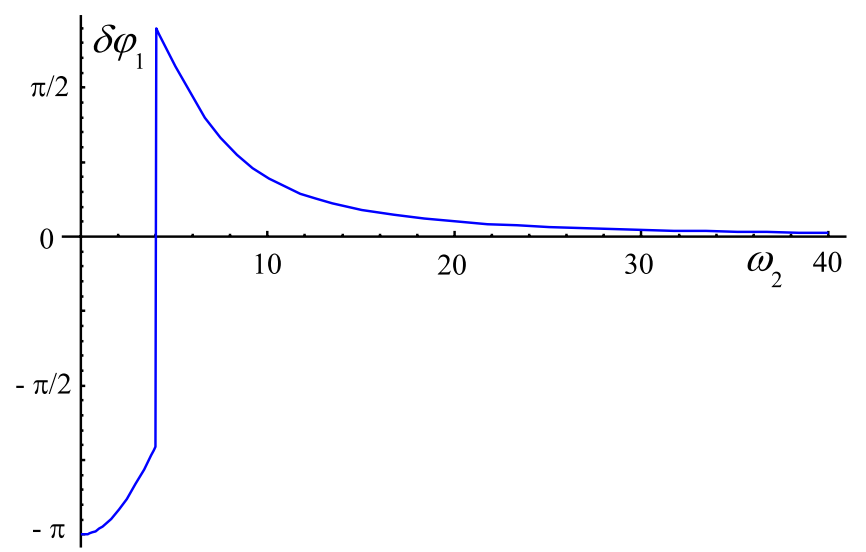

FIG. 5. (Color online) The phase shift $\delta_{\varphi_{1}}$ of FCP 1 against the frequency $\omega_{2}$ of FCP 2 . The discontinuity corresponds to the equality of the group velocities of the two FCPs. Parameters: $a=b=1$, $p_{1}=p_{2}=2$, and $\omega_{1}=4$.
The phase shift of the FCP 2 is then obtained from Eq. (25) by permuting the indices 1 and 2 . As in the case of the location shift $\delta_{\tau_{1}}$, the phase shift $\delta_{\varphi_{1}}$ does not depend on the coefficients $a$ and $b$ of the $\mathrm{mKdV}$-sG equation, except its sign, via the group velocities. Examples of variation of the phase shift against the frequency of one of the FCPs are shown in Figs. 5 and 6. Again, it is seen that no phase matching is required, and the shift vanishes in the SVEA limit. However, if $p_{1}$ tends to zero, which corresponds to a pulse 1 in the SVEA limit interacting with FCP 2, the phase shift $\delta_{\varphi_{1}}$ reaches a nonzero limit: the interaction with an FCP may affect the phase of a pulse that is not extremely short.

\section{CONCLUSION}

In conclusion, we have studied the interactions between few-cycle optical solitons by using the known two-breather solutions [19] of the mKdV-sG equation. The shapes of both 
input and output soliton envelopes as well as the phase and location shifts have been computed by using the exact expressions for the four-soliton (two-breather) solutions of the $\mathrm{mKdV}-\mathrm{sG}$ equation. The remarkable fact is that, in contrast with the case of soliton envelopes, no phase matching of any kind is required for two few-cycle pulses to interact efficiently. Such interactions may be a problem for the realization and propagation of a regular train of few-cycle pulses.
[1] T. Brabec and F. Krausz, Rev. Mod. Phys. 72, 545 (2000).

[2] M. Wegener, Extreme Nonlinear Optics (Springer-Verlag, Berlin, 2005).

[3] A. Scrinzi, M. Yu. Ivanov, R. Kienberger, and D. M. Villeneuve, J. Phys. B 39, R1 (2006).

[4] T. Brabec and F. Krausz, Phys. Rev. Lett. 78, 3282 (1997).

[5] M. V. Tognetti and H. M. Crespo, J. Opt. Soc. Am. B 24, 1410 (2007).

[6] T. Schäfer and C. E. Wayne, Physica D 196, 90 (2004).

[7] A. Sakovich and S. Sakovich, J. Phys. A 39, L361 (2006).

[8] S. A. Skobelev, D. V. Kartashov, and A. V. Kim, Phys. Rev. Lett. 99, 203902 (2007); A. V. Kim, S. A. Skobelev, D. Anderson, T. Hansson, and M. Lisak, Phys. Rev. A 77, 043823 (2008).

[9] I. V. Mel'nikov, D. Mihalache, F. Moldoveanu, and N.-C. Panoiu, Phys. Rev. A 56, 1569 (1997).

[10] H. Leblond and F. Sanchez, Phys. Rev. A 67, 013804 (2003).

[11] I. V. Mel'nikov, H. Leblond, F. Sanchez, and D. Mihalache, IEEE J. Sel. Top. Quantum Electron. 10, 870 (2004).
[12] H. Leblond, S. V. Sazonov, I. V. Mel'nikov, D. Mihalache, and F. Sanchez, IEEE J. Sel. Top. Quantum Electron. 74, 063815 (2006)

[13] R. K. Dodd, J. C. Eilbeck, J. D. Gibbon, and H. C. Morris, Solitons and Nonlinear Wave Equations (Academic, London, 1982).

[14] K. Konno, W. Kameyama, and H. Sanuki, J. Phys. Soc. Jpn. 37, 171 (1974).

[15] I. V. Mel'nikov, D. Mihalache, and N.-C. Panoiu, Opt. Commun. 181, 345 (2000).

[16] H. Leblond, F. Sanchez, I. V. Mel'nikov, and D. Mihalache, Opt. Commun. 69, 378 (2005).

[17] H. Leblond, Phys. Rev. A 78, 013807 (2008).

[18] X. Tan, X. Fan, Y. Yang, and D. Tong, J. Mod. Opt. 55, 2439 (2008).

[19] D.-Y. Chen, D.-J. Zhang, and S.-F. Deng, J. Phys. Soc. Jpn. 71, 658 (2002). 